\title{
On the number of two-dimensional threshold functions
}

\author{
Max A. Alekseyev \\ $\langle$ maxal@cse.sc.edu〉 \\ Department of Computer Science and Engineering \\ University of South Carolina, U.S.A.
}

March 5, 2022

\begin{abstract}
A two-dimensional threshold function of $k$-valued logic can be viewed as coloring of the points of a $k \times k$ square lattice into two colors such that there exists a straight line separating points of different colors. For the number of such functions only asymptotic bounds are known. We give an exact formula for the number of two-dimensional threshold functions and derive more accurate asymptotics.
\end{abstract}


The number of two-dimensional threshold functions

\section{Introduction}

A function $f$ of $n$ variables of $k$-valued logic is called a threshold function if it takes two values 0 and 1 and there exists a hyperplane separating the pre-images $f^{-1}(0)$ and $f^{-1}(1)$. Threshold functions have been studied from the perspectives of electrical engineering [6], neural networks [3], combinatorial geometry [1, 8, 4], and learning theory [11, 15].

Computing the number $P(k, n)$ of $n$-dimensional $k$-valued threshold functions turns out to be a hard problem, even in the case of $k=2$. The number $P(2, n)$, corresponding to $n$-dimensional boolean threshold functions, was studied in a number of publications [14, 7, 8, 18]. In spite of many efforts, the exact values of $P(2, n)$ are known only for $n \leq 8$ (sequence $A 000609$ in [12]). The asymptotics of $P(2, n)$ was found in [16, 17]. Computing $P(k, n)$ for $k>2$ appears to be even a harder problem. Known results on the number $P(k, n)$ for $k>2$ are mostly of an asymptotic nature [5, 4]. A particular case of twodimensional threshold functions (i.e., for $n=2$ ) was studied in [11]. Shevchenko [10] states the following asymptotic bounds

$$
\frac{3}{8 \pi^{2}} k^{4} \lesssim P(k, 2) \lesssim \frac{6}{\pi^{2}} k^{4} .
$$

In this paper we prove (in Theorem 3 ) an exact formula for $P(k, 2):{ }_{1}^{1}$

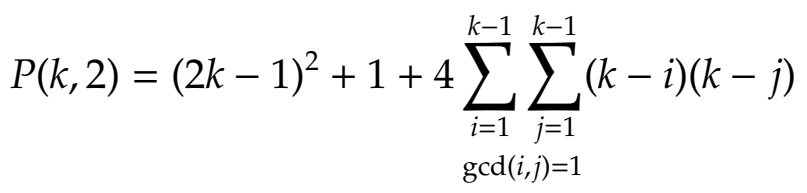

and derive (in Theorem 25) a more accurate asymptotics:

$$
P(k, 2)=\frac{6}{\pi^{2}} k^{4}+O\left(k^{3} \ln k\right) .
$$

The paper is organized as follows. In Section 2 we give a rigorous definition and examples of two-dimesional threshold functions. The exact formula for $P(k, 2)$ is obtained in Section 3. The asymptotics for $P(k, 2)$ is derived in Section 4 . Finally, in Section 5 we discuss the connection to teaching sets and pose a related open problem.

\section{Two-dimensional threshold functions}

We consider the problem of finding $P(k, 2)$ and its asymptotics in a slightly more general form, allowing the arguments of two-dimensional threshold functions take different numbers of integer values. The precise definitions follow:

Definition 1. Let $m, n \in \mathbb{N}$ be positive integers and $\mathcal{K} \stackrel{\text { def }}{=}[0, m] \times[0, n]$ be a rectangle on Euclidean plane bounded by the lines $x=0, x=m, y=0$, and $y=n$. The four sides of $\mathcal{K}$ form its border and a point in $\mathcal{K}$ is called internal if it does not belong to the border of $\mathcal{K}$. We further define $\mathcal{K}_{0} \stackrel{\text { def }}{=} \mathcal{K} \cap \mathbb{Z}^{2}$ as the set of all integer points in $\mathcal{K}$.

\footnotetext{
${ }^{1}$ The values of $P(k, 2)$ and $\frac{1}{2} P(k, 2)$ for $k=1,2,3, \ldots$ form respectively the sequences $A 114146$ and $A 114043$ in [12].
} 
a)

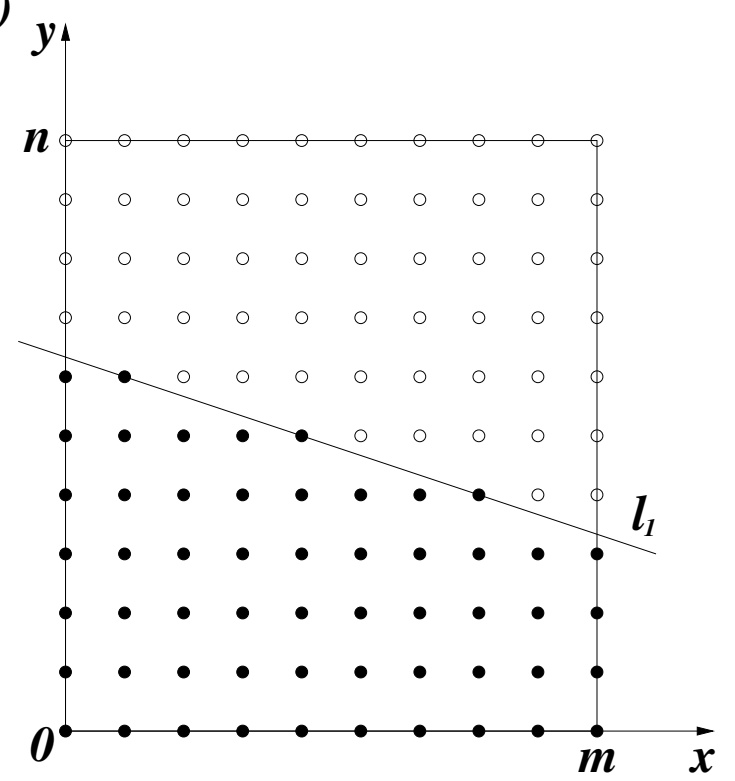

b)

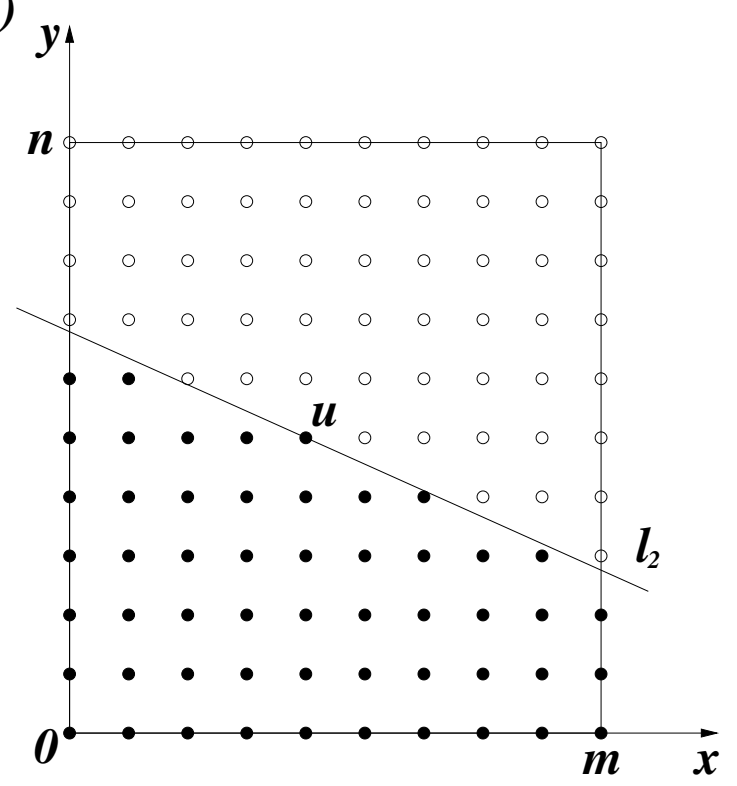

Figure 1: Examples of threshold functions defined by the lines $\ell_{1}$ and $\ell_{2}$ (the filled dots correspond to zero values). a) The line $\ell_{1}$ defines a stable threshold function. b) The line $\ell_{2}$ defines an unstable threshold function with the vertex at $u$.

Definition 2. $A$ (two-dimensional) threshold function on $\mathcal{K}_{0}$ is a function $f: \mathcal{K}_{0} \rightarrow\{0,1\}$ such that there exists a line $\ell=a x+b y+c \equiv 0$ satisfying

$$
f(x, y)=0 \quad \Longleftrightarrow \quad a x+b y+c \leq 0 .
$$

We say that the line $\ell$ defines the threshold function $f$.

Examples of threshold functions are given in Fig. 1.

Let $N(m, n)$ be the number of all threshold functions on $\mathcal{K}_{0}$. Our goal is to find an exact formula and an asymptotics for $N(m, n)$. That will immediately imply similar results for $P(k, 2)$ since

$$
P(k, 2)=N(k-1, k-1) .
$$

\section{Exact formula for $N(m, n)$}

In this section we prove the following theorem, which together with (3) implies formula (1).

Theorem 3. The total number of two-dimensional threshold functions is

$$
N(m, n)=(2 m+1)(2 n+1)+1+4 V(m, n),
$$

where

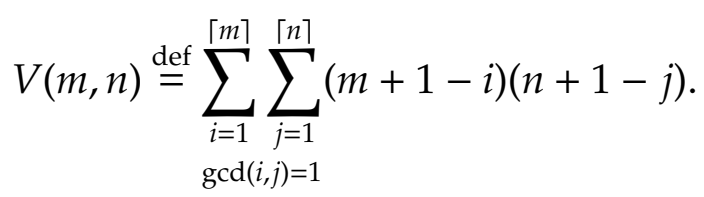


The number of two-dimensional threshold functions

\subsection{Preliminary results}

All threshold functions fall into two classes, depending on the value of $f(0,0)$. Between these two classes there is a natural bijection $f \mapsto 1-f$. Let $\mathcal{F}$ be a class of threshold functions with $f(0,0)=0$, excluding the zero function. Then the total number of threshold functions equals $2(|\mathcal{F}|+1)$.

With each line $\ell=a x+b y+c \equiv 0$ we associate a set of zeros of a threshold function defined by $\ell$ :

$$
M(\ell)=M(a, b, c)=\left\{(x, y) \in \mathcal{K}_{0} \mid a x+b y+c \leq 0\right\} .
$$

If the line $\ell$ defines a function from $\mathcal{F}$, then $c \leq 0$. For the rest, we assume that this inequality always holds.

We note that $M(\ell)$ is well-defined only for a line $\ell=a x+b y+c \equiv 0$ with $c \neq 0$ (a regular line); however, a line with $c=0$ (a singular line) generally defines two threshold functions corresponding to $M(a, b, 0)$ and $M(-a,-b, 0)$. For a singular line $\ell^{\prime}$ resulting from continuous motion of a regular line $\ell$, we assume that $\ell^{\prime}$ defines a function equal the limit of the function defined by $\ell$.

Definition 4. Call a line $\ell=a x+b y+c \equiv 0$ horizontal, if $a=0$; vertical, if $b=0$; and inclined, if $a \neq 0$ and $b \neq 0$. An inclined line is called positive or negative depending on the sign of its slope $\frac{-a}{b}$.

Definition 5. Lines $\ell_{1}$ and $\ell_{2}$ are equivalent $\left(\ell_{1} \sim \ell_{2}\right)$, if $M\left(\ell_{1}\right)=M\left(\ell_{2}\right)$. In other words, two lines are equivalent if they define the same threshold function.

We prove (non-)equivalence of the lines $\ell_{1}$ and $\ell_{2}$ using (non-)emptiness of the symmetric difference $M\left(\ell_{1}\right) \triangle M\left(\ell_{2}\right)$, or both set differences $M\left(\ell_{1}\right) \backslash M\left(\ell_{2}\right)$ and $M\left(\ell_{2}\right) \backslash M\left(\ell_{1}\right)$.

Lemma 6. For any line $\ell$ defining a threshold function from $\mathcal{F}$, there exists an equivalent line $\ell^{\prime}$ passing through at least one point from $\mathcal{K}_{0}$.

Proof. Let the line $\ell=a x+b y+c \equiv 0$ define some threshold function from $\mathcal{F}$. If $\ell$ does not contain points from $\mathcal{K}_{0}$, then increasing $c$ (i.e., translating $\ell$ towards the origin) we will find a line $\ell^{\prime}=a x+b y+c^{\prime} \equiv 0$ such that $\ell^{\prime}$ passes through at least one point from $\mathcal{K}_{0}$, and there are no points from $\mathcal{K}_{0}$ between $\ell^{\prime}$ and $\ell$. Then $\ell \sim \ell^{\prime}$.

Denote by $\mathfrak{Q}$ the set of all lines that define functions from $\mathcal{F}$ and pass through at least one point from $\mathcal{K}_{0}$. Lemma 6 implies that every function from $\mathcal{F}$ is defined by some line from $\mathbb{R}$.

Lemma 7. Let $\ell_{1}, \ell_{2} \in \mathbb{R}$. If $\ell_{1} \sim \ell_{2}$ and $\ell_{1} \neq \ell_{2}$, then there exists a point $q \in \ell_{1} \cap \ell_{2} \cap \mathcal{K}$.

Proof. Assume that the lines $\ell_{1}$ and $\ell_{2}$ do not have a common point within the rectangle $\mathcal{K}$. Since $\ell_{1}, \ell_{2} \in \mathfrak{L}$, there exist points $u \in \ell_{1} \cap \mathcal{K}_{0}$ and $v \in \ell_{2} \cap \mathcal{K}_{0}$. Then either $u \in M\left(\ell_{1}\right) \backslash M\left(\ell_{2}\right)$ or $v \in M\left(\ell_{2}\right) \backslash M\left(\ell_{1}\right)$, a contradiction to $\ell_{1} \sim \ell_{2}$.

Definition 8. A line $\ell \in \mathfrak{Q}$ is called stable if it passes through at least two points from $\mathcal{K}_{0}$.

An example of a stable line is given in Fig. 17. 
Lemma 9. Let $\ell_{1} \sim \ell_{2}$ be equivalent lines passing through points $u \in \mathcal{K}_{0}$ and $v \in \mathcal{K}_{0}$ respectively. If $u \neq v$ then there exists a stable line $\ell_{0}$ such that $u \in \ell_{0}$ and $\ell_{1} \sim \ell_{0} \sim \ell_{2}$.

Proof. If $\ell_{1}$ is stable, then the statement is trivial for $\ell_{0}=\ell_{1}$. Assume that $\ell_{1}$ is not stable.

Lemma 7 implies $\ell_{1} \cap \ell_{2}=q \in \mathcal{K}$. Since $\ell_{1}$ is not stable, $q \neq v$. On the other hand, if $q=u$ then $\ell_{0}=\ell_{2}$ proves the lemma. Hence, assume $q \neq u$.

Consider a family of lines $\ell(t)$ passing through the points $u$ and $q+(v-q) t$ for $t \in[0,1]$. Note that $\ell(0)=\ell_{1}$. Let $t_{0}>0$ be a minimal value of $t$ such that line $\ell(t)$ passes through a point from $\mathcal{K}_{0}$ different from $u$. Define $\ell_{0}=\ell\left(t_{0}\right)$.

We will show that the set $M(\ell(t))$ does not change as the parameter $t$ changes from 0 to $t_{0}$. By the construction, $M\left(\ell^{\prime}\right)=M\left(\ell_{1}\right)$ for any intermediate line $\ell^{\prime}=\ell(t), t \in\left(0, t_{0}\right)$ and all the points from $\ell_{1} \cap \mathcal{K}_{0}$, except $u$, lie on the same side of $\ell^{\prime}$ as the point $v \in M\left(\ell_{1}\right)=M\left(\ell_{2}\right)$. Hence, $M\left(\ell^{\prime}\right)=M\left(\ell_{1}\right)$. On the other hand, for any point $w \in \ell_{0} \cap \mathcal{K}_{0}$, it is true that $w \in M\left(\ell_{1}\right)=M\left(\ell_{2}\right)$. Indeed, if $w \notin M\left(\ell_{1}\right)=M\left(\ell_{2}\right)$, then the point $w$ must lie on a ray of $\ell_{0}$ starting at $u$ that crosses the line $\ell_{2}$. But then the points $w$ and $v$ lie at the same side of line $\ell_{1}$, and thus $w \in M\left(\ell_{1}\right)$ which contradicts the assumption $w \notin M\left(\ell_{1}\right)=M\left(\ell_{2}\right)$.

Therefore, $M\left(\ell_{0}\right)=M\left(\ell\left(t_{0}\right)\right)=M(\ell(0))=M\left(\ell_{1}\right)$, i. e., $\ell_{0} \sim \ell_{1}$.

Lemma 10. If equivalent stable lines $\ell_{1}$ and $\ell_{2}$ intersect at a point from $\mathcal{K}_{0}$, then $\ell_{1}=\ell_{2}$.

Proof. Let $\ell_{1} \cap \ell_{2}=u \in \mathcal{K}_{0}$. Suppose that the line $\ell_{1}$ passes through points $u \neq v \in \mathcal{K}_{0}$, while the line $\ell_{2}$ passes through points $u \neq w \in \mathcal{K}_{0}$.

If the line $\ell_{1}$ is vertical, then $u_{x}=v_{x}=c$. In this case the line $\ell_{2}$ cannot be horizontal, since otherwise the corresponding threshold function would be the zero function which is not in $\mathcal{F}$. From $w \in M\left(\ell_{2}\right)=M\left(\ell_{1}\right)$ it follows that $w_{x} \leq c$. If $w_{x}<c$, then $\left(c, w_{y}\right) \in$ $M\left(\ell_{1}\right) \backslash M\left(\ell_{2}\right)$ which contradicts $\ell_{1} \sim \ell_{2}$. Therefore, $w_{x}=c$ and $\ell_{2}=\ell_{1}$.

The other cases with a horizontal or vertical line are considered similarly. Assume that both lines $\ell_{1}, l_{2}$ are inclined.

If the point $u$ lies on the border of $\mathcal{K}$, then either $v \notin M\left(\ell_{2}\right)$ or $w \notin M\left(\ell_{1}\right)$, a contradiction to $\ell_{1} \sim \ell_{2}$. Hence, $u$ is an internal point of the rectangle $\mathcal{K}$.

It is easy to see that if the lines $\ell_{1}$ and $\ell_{2}$ have opposite signs, then the line $x=u_{x}$ or $y=u_{y}$ contains a point from $M\left(\ell_{1}\right) \triangle M\left(\ell_{2}\right)$.

The remaining case to consider is the lines $\ell_{1}$ and $\ell_{2}$ having the same sign. Without loss of generality, assume that they are positive. Then each of them crosses the left or bottom side of the rectangle $\mathcal{K}$. Note that the case, when one line crosses the left side while the other crosses the bottom side, is impossible since it would imply $(0, n) \in M\left(\ell_{1}\right) \triangle M\left(\ell_{2}\right)$ which contradicts $\ell_{1} \sim \ell_{2}$. Without loss of generality, assume that both lines cross the bottom side of $\mathcal{K}$.

Suppose that the slopes of $\ell_{1}$ and $\ell_{2}$ are not equal. Without loss of generality, assume that the slope of $\ell_{1}$ is less than the slope of $\ell_{2}$. Then $v_{x}<u_{x}<w_{x}$ and $v_{y}<u_{y}<w_{y}$. Hence, line $\ell$, passing through the points $v$ and $w$, is positive (Fig. 2 a).

Let $z=\frac{v+w}{2}$ be the middle point of the interval $[v, w]$. Consider the point $v^{\prime}$ symmetric to $v$ with respect to the point $u$. It is easy to see that $v^{\prime} \notin \mathcal{K}_{0}$, since otherwise $v^{\prime} \in M\left(\ell_{1}\right) \backslash M\left(\ell_{2}\right)$ contradicts $\ell_{1} \sim \ell_{2}$. In particular, we have $v_{x}^{\prime}=2 u_{x}-v_{x}>w_{x}$, which is equivalent to $u_{x}>z_{x}$. Similarly, for the point $w^{\prime}$ symmetric to $w$ with respect to $u$, we have $u_{y}<z_{y}$. Since $u$ is an 

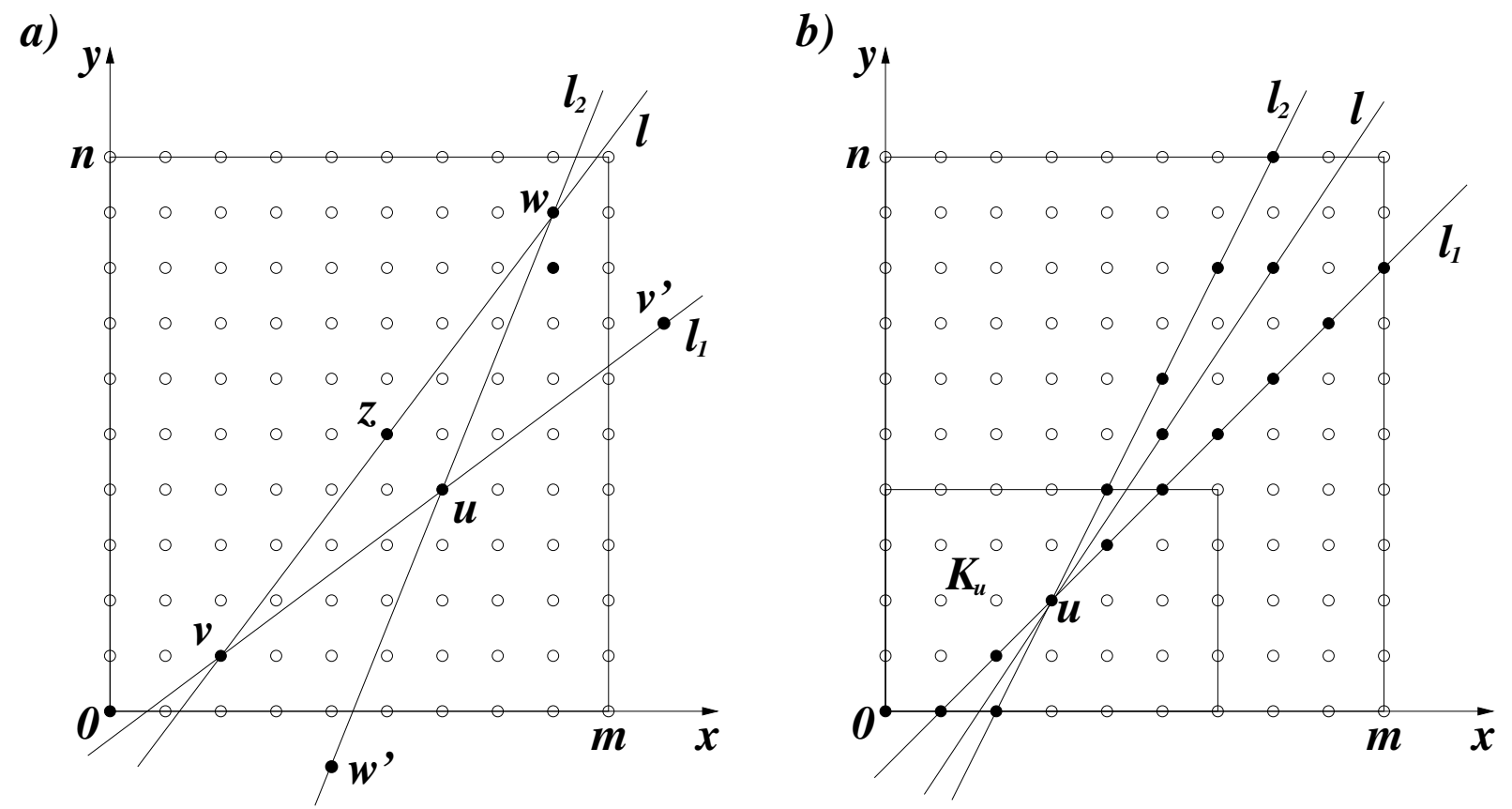

Figure 2: a) The point $\left(w_{x}, w_{y}-1\right)$ belongs to $M\left(\ell_{1}\right) \backslash M\left(\ell_{2}\right)$, implying that $\ell_{1}$ and $\ell_{2}$ are not equivalent. b) The line $\ell$ lies between the adjacent $u$-stable lines $\ell_{1}$ and $\ell_{2}$. The line $\ell$ is stable but not $u$-stable since it has no integer points within the rectangle $K_{u}$, except $u$.

integral point and $z$ is the middle point of the interval with integral endpoints, a stronger inequality $z_{y}-u_{y} \geq \frac{1}{2}$ holds.

Consider an equation of the line $\ell_{1}$ in the form $y_{1}(x)=\frac{u_{y}-v_{y}}{u_{x}-v_{x}}\left(x-v_{x}\right)+v_{y}$ and an equation of the line $\ell$ in the form $y(x)=\frac{w_{y}-v_{y}}{w_{x}-v_{x}}\left(x-v_{x}\right)+v_{y}$. Define a function

$$
f(x) \stackrel{\text { def }}{=} y\left(x+v_{x}\right)-y_{1}\left(x+v_{x}\right)=\left(\frac{w_{y}-v_{y}}{w_{x}-v_{x}}-\frac{u_{y}-v_{y}}{u_{x}-v_{x}}\right) x .
$$

Trivially, we have $f(k x)=k f(x)$ for all $k$.

Since the line $\ell_{1}$ is positive, and $z_{x}<u_{x}$, then $y_{1}\left(z_{x}\right)<y_{1}\left(u_{x}\right)=u_{y}$. Hence, $f\left(\frac{w_{x}-v_{x}}{2}\right)=$ $z_{y}-y_{1}\left(z_{x}\right)>z_{y}-u_{y} \geq \frac{1}{2}$. Linearity of $f(x)$ implies

$$
w_{y}-y_{1}\left(w_{x}\right)=f\left(w_{x}-v_{x}\right)=2 f\left(\frac{w_{x}-v_{x}}{2}\right)>1,
$$

which is equivalent to $y_{1}\left(w_{x}\right)<w_{y}-1$. Hence, $\left(w_{x}, w_{y}-1\right) \in M\left(\ell_{1}\right) \backslash M\left(\ell_{2}\right)$ which contradicts $\ell_{1} \sim \ell_{2}$. The contradiction proves that the slopes of $\ell_{1}$ and $\ell_{2}$ are equal. Since $\ell_{1}$ and $\ell_{2}$ have the common point $u$, Lemma 10 implies $\ell_{1}=\ell_{2}$.

Lemma 11. If lines $\ell_{1}$ and $\ell_{2}$ are stable and $\ell_{1} \sim \ell_{2}$, then $\ell_{1}=\ell_{2}$.

Proof. Assume that lines $\ell_{1}$ and $\ell_{2}$ are stable and $\ell_{1} \sim \ell_{2}$, but $\ell_{1} \neq \ell_{2}$. By Lemma 7 . $\ell_{1} \cap \ell_{2}=q \in \mathcal{K}$. 
If $q \in \mathcal{K}_{0}$, then Lemma 10 implies $\ell_{1}=\ell_{2}$, a contradiction.

Assume $q \notin \mathcal{K}_{0}$. Let points $u, v \in \mathcal{K}_{0}$ be the closest to $q$ lying on the lines $\ell_{1}$ and $\ell_{2}$ respectively. By Lemma 9 there exists a stable line $\ell_{0}$ equivalent to $\ell_{1}$ such that $u \in \ell_{0}$. Lemma 10 applied to the lines $\ell_{0}$ and $\ell_{1}$ implies $\ell_{0}=\ell_{1}$, which is impossible since $\ell_{0}$ differs from $\ell_{1}$ by construction. This contradiction completes the proof.

Lemma 12. Let the line $\ell_{1}$ pass through a point $u \in \mathcal{K}_{0}$. If line $\ell_{2}$ is stable and $\ell_{1} \sim \ell_{2}$, then $\ell_{2}$ passes through $u$ as well.

Proof. If the line $\ell_{1}$ is stable, then the statement immediately follows from Lemma 10 Hence, suppose that the line $\ell_{1}$ is not stable, i.e., $\ell_{1} \cap \mathcal{K}_{0}=\{u\}$.

According to Lemma 7, $\ell_{1} \cap \ell_{2}=q \in \mathcal{K}$. Let $v \in \mathcal{K}_{0}$ be a point on the line $\ell_{2}$ closest to the point $q$.

If $q \in \mathcal{K}_{0}$, i.e., $v=u=q$, then the lemma is proved.

Assume $q \notin \mathcal{K}_{0}$. By Lemma 9 , there exists a stable line $\ell_{0}$ such that $u \in \ell_{0}$ and $\ell_{0} \sim \ell_{1}$. Applying Lemma 11 to the lines $\ell_{0}$ and $\ell_{2}$, we conclude that $\ell_{0}=\ell_{2}$ and thus $u \in \ell_{2}$.

Definition 13. A threshold function $f \in \mathcal{F}$ is called stable, if there exists a stable line that defines $f$. If there is no such line, the function $f$ is called unstable.

Examples of stable and unstable functions are given in Fig. 1.

\subsection{Number of unstable functions}

Definition 14. Lemma 9 implies that for an unstable function $f$, every line from $\mathbb{Q}$ defining $f$ passes through the very same point from $K_{0}$. We call this point the vertex of $f$.

Definition 15. For $u \in \mathcal{K}_{0}$, let $\mathcal{K}_{u}$ be the largest axis-parallel rectangle contained in $\mathcal{K}$ with the center at $u$. Further let $\overline{\mathcal{K}_{u}}=\mathcal{K} \backslash \mathcal{K}_{u}$. Denote by $L_{u}$ the set of all lines passing through $u$ and by $S_{u}$ the set of all stable lines from $L_{u}$. A line $\ell$ is called $u$-stable if $\ell \in S_{u}$ and it passes through a point from $\left(\mathcal{K}_{u} \cap \mathcal{K}_{0}\right) \backslash\{u\}$.

Definition 16. Let $u \in \mathcal{K}_{0}$. Lines $\ell_{1}, \ell_{2} \in S_{u}$ are called adjacent if in the shortest rotation of a line about the point $u$ from the position $\ell_{1}$ to the position $\ell_{2}$, it does not meet any other lines from $S_{u}$. Any intermediate line in this rotation is said to be lying between the lines $\ell_{1}$ and $\ell_{2}$ (Fig. $2 p$ ).

Lemma 17. If a line $\ell$ passing through $u \in \mathcal{K}_{0}$ lies between adjacent lines $\ell_{1}, \ell_{2} \in S_{u}$, and $\ell \sim \ell^{\prime}$ for some stable line $\ell^{\prime}$, then either $\ell^{\prime}=\ell_{1}$ or $\ell^{\prime}=\ell_{2}$.

Proof. Assume that $\ell^{\prime}$ differs from $\ell_{1}$ and $\ell_{2}$. Let $v_{1}$ and $v_{2}$ be points from $\mathcal{K}_{0}$ different from $u$ lying on the lines $\ell_{1}$ and $\ell_{2}$ respectively. Then Lemma 12 implies that $u \in \ell^{\prime}$. In the shortest rotation about the point $u$ towards the line $\ell^{\prime}$, the line $\ell$ necessarily meets $\ell_{t}$ for $t=1$ or 2 . But then the point $v_{t}$ belongs to $M(\ell) \Delta M\left(\ell^{\prime}\right)$ and thus $\ell$ and $\ell^{\prime}$ cannot be equivalent. This contradiction completes the proof.

For $u=0$, there exists a unique unstable threshold function with the vertex at $u$. Namely, this function takes value 0 only at the point 0 and thus is defined by any negative singular line. 
If $u \neq 0$ is a corner vertex of the rectangle $\mathcal{K}$, then there is no unstable threshold function with the vertex at $u$. It is easy to see that any line passing through $u$ can be rotated about $u$ into an equivalent stable line.

Denote by $\mathcal{K}_{0}^{\prime}$ the set $\mathcal{K}_{0}$ with excluded the corner vertices of the rectangle $\mathcal{K}$.

Lemma 18. Let $u \in \mathcal{K}_{0}^{\prime}$, and $\ell_{0}$ be a line passing through the points $\mathbf{0}$ and $u$. Then the number of unstable threshold functions with the vertex at $u$ is equal to the number of $u$-stable lines, if the line $\ell_{0}$ is $u$-stable; and one less otherwise.

Proof. Suppose that a line $\ell \in L_{u} \backslash S_{u}$ lies between adjacent lines $\ell_{1}, \ell_{2} \in S_{u}$. Note that $\ell$ cannot be equivalent to any $u$-stable line, since the latter contains a pair of symmetric (with respect to $u$ ) points, one of which does not belong to $M(\ell)$. Hence, if both lines $\ell_{1}$ and $\ell_{2}$ are $u$-stable, then $\ell$ is not equivalent to either of them and thus by Lemma 17defines an unstable threshold function.

Therefore, the statement is true when $\mathcal{K}_{u}=\mathcal{K}$ (e.g., $u$ is the center of the rectangle $\left.\mathcal{K}\right)$. For the rest of the proof, we assume that $u$ is not the center of $\mathcal{K}$.

Let us split the set $L_{u}$ into two:

$$
\begin{aligned}
& L_{u}^{\prime} \stackrel{\text { def }}{=}\left\{\ell \in L_{u} \mid \ell \cap \overline{\mathcal{K}_{u}}=\emptyset\right\} ; \\
& L_{u}^{\prime \prime} \stackrel{\text { def }}{=}\left\{\ell \in L_{u} \mid \ell \cap \overline{\mathcal{K}_{u}} \neq \emptyset\right\} .
\end{aligned}
$$

Any unstable line from $L_{u}^{\prime}$ lies between two adjacent stable lines from $L_{u}^{\prime}$, which are trivially $u$-stable as well. Hence, the number of distinct unstable threshold functions defined by lines from $L_{u}^{\prime}$ equals the number of $u$-stable lines in $L_{u}^{\prime}$ minus 1 .

Any unstable line $\ell \in L_{u}^{\prime \prime}$ consists of two rays starting at $u$ such that one of them crosses $\overline{\mathcal{K}_{u}}$. Consider a rotation of $\ell$ about $u$ such this ray moves towards the origin, following the shortest arc. Without loss of generality, we assume that in this rotation, the line $\ell$ first meets the stable line $\ell_{1}$. Then $\ell$ cannot be equivalent to the line $\ell_{2}$, since $\ell_{2}$ is either $u$-stable or passes through a point from $\overline{\mathcal{K}_{u}} \cap \mathcal{K}_{0}$ not belonging to $M(\ell)$. On the other hand, $\ell$ is equivalent to $\ell_{1}$ if and only if $\ell_{1}$ is not $u$-stable. Hence, the counting of unstable threshold functions with the vertex at $u$ corresponds to counting of $u$-stable lines. The number of unstable threshold functions defined by lines from $L_{u}^{\prime \prime}$ equals the number of $u$-stable lines in $L_{u}^{\prime \prime}$.

Note that if the line $\ell_{0}$ is $u$-stable, it is counted two times. In this case, the number of unstable threshold functions defined by lines from $L_{u}^{\prime \prime}$ is greater by 1 as compared to the number of $u$-stable lines in $L_{u}^{\prime \prime}$.

Therefore, the total number of unstable threshold functions with the vertex at $u$ is equal to the number of $u$-stable lines if the line $\ell_{0}$ is $u$-stable; and is less by 1 otherwise.

Definition 19. Let

$$
U(p, q) \stackrel{\text { def }}{=} \#\left\{(a, b) \in \mathbb{Z}^{2} \mid 1 \leq a \leq p, 1 \leq b \leq q, \operatorname{gcd}(a, b)=1\right\} .
$$

In other words,

$$
U(p, q)=\sum_{\substack{i=1 \\ \operatorname{gcd}(i, j)=1}}^{p} \sum_{j=1}^{q} 1 .
$$


It is easy to see that $U(m, n)$ gives the number of singular inclined stable lines, each defined by the slope $\frac{b}{a}$ (i.e., passing through the points 0 and $(a, b)$ ) with $1 \leq a \leq m$, $1 \leq b \leq n$, and $\operatorname{gcd}(a, b)=1$. We also remark that the values $U(k, k)$ are related to the probability of two random integers from $[1, k]$ being co-prime (see sequence $A 018805$ in [12]).

Lemma 20. The number of points $u \in \mathcal{K}_{0}^{\prime}$ such that the line passing through the points $\mathbf{0}$ and $u$ is not $u$-stable equals $U(m, n)-1$.

Proof. If a line $\ell$ passes through exactly $k+1$ points $\mathbf{0}=u_{0}, u_{1}, \ldots, u_{k} \in \mathcal{K}_{0}$ listed in the order of increasing distance from the point 0 , then $\ell$ is $u_{i}$-stable for $i=1,2, \ldots, k-1$ and is not $u_{k}$-stable. Therefore, on each inclined singular stable line $\ell$ there is exactly one point $u \in \mathcal{K}_{0}$ such that $\ell$ is not $u$-stable. Hence, the number of such points $u \in \mathcal{K}_{0}^{\prime}$ equals the number of inclined singular stable lines, excluding the line $y=\frac{n}{m} x$ with $u=(m, n) \notin \mathcal{K}_{0}^{\prime}$, which is $U(m, n)-1$.

Definition 21. For real numbers $t$ and $k$, define a function

$$
V(t, k) \stackrel{\text { def }}{=} \sum_{\substack{i=1 \\ \operatorname{gcd}(i, j)=1}}^{\lceil k\rceil}(t+1-i)(k+1-j) .
$$

Lemma 22. For all $t \in \mathbb{R}, k \in \mathbb{N}$ the following equality holds

$$
V(t, k-1)+V(t, k)=2 V\left(t, k-\frac{1}{2}\right) .
$$

For all $t, k \in \mathbb{N}$ the following equalities hold

$$
\begin{gathered}
V(t, k)=\sum_{p=1}^{t} \sum_{q=1}^{k} U(p, q) ; \\
\sum_{p=1}^{t} U(p, k)=V(t, k)-V(t, k-1) ; \\
U(t, k)=V(t, k)-V(t, k-1)-V(t-1, k)+V(t-1, k-1) ; \\
U(t, k)+2(V(t, k-1)+V(t-1, k))=4 V\left(t-\frac{1}{2}, k-\frac{1}{2}\right) .
\end{gathered}
$$

Proof. Let $t \in \mathbb{R}, k \in \mathbb{N}$. To prove (6), we use formula (5)

$$
\begin{aligned}
V(t, k-1)+V(t, k) & =\sum_{\substack{i=1 \\
\operatorname{gcd}(i, j)=1}}^{\lceil t\rceil} \sum_{j=1}^{k-1}(t+1-i)(k-j)+\sum_{\substack{i=1 \\
\operatorname{gcd}(i, j)=1}}^{[t]} \sum_{\substack{j=1\\
}}^{k}(t+1-i)(k+1-j) \\
& =\sum_{\substack{i=1 \\
\operatorname{gcd}(i, j)=1}}^{k} \sum_{j=1}^{[t]}(t+1-i)(2 k+1-2 j)=2 \sum_{\substack{i=1 \\
\operatorname{gcd}(i, j)=1}}^{k}(t+1-i)\left(k+\frac{1}{2}-j\right) \\
& =2 V\left(t, k-\frac{1}{2}\right) .
\end{aligned}
$$


The number of two-dimensional threshold functions

To prove (7), we use formulae (4) and (5)

$$
\begin{aligned}
& \sum_{p=1}^{t} \sum_{q=1}^{k} U(p, q)=\sum_{p=1}^{t} \sum_{q=1}^{k} \sum_{\substack{i=1 \\
\operatorname{gcd}(i, j)=1}}^{p} \sum_{\substack{j=1 \\
\operatorname{gcd}(i, j)=1}}^{q} 1=\sum_{\substack{i=1 \\
y_{1}}}^{k} \sum_{p=j}^{t} \sum_{\substack{i=1 \\
\operatorname{gcd}(i, j)=1}}^{k} 1=\sum_{j=1}^{k}(t+1-i)(k+1-j) \\
& =V(t, k) \text {. }
\end{aligned}
$$

To prove (8), we use formula (7)

$$
V(t, k)-V(t, k-1)=\sum_{p=1}^{t} \sum_{q=1}^{k} U(p, q)-\sum_{p=1}^{t} \sum_{q=1}^{k-1} U(p, q)=\sum_{p=1}^{t} U(p, k) .
$$

Using (8), we prove (9)

$$
U(t, k)=\sum_{p=1}^{t} U(p, k)-\sum_{p=1}^{t-1} U(p, k)=V(t, k)-V(t, k-1)-V(t-1, k)+V(t-1, k-1) .
$$

Finally, to prove equality (10), we use formulae (9) and (6)

$$
\begin{aligned}
U(t, k)+2(V(t, k-1)+V(t-1, k)) & =V(t, k)+V(t, k-1)+V(t-1, k)+V(t-1, k-1) \\
& =2 V\left(t, k-\frac{1}{2}\right)+2 V\left(t-1, k-\frac{1}{2}\right) \\
& =4 V\left(t-\frac{1}{2}, k-\frac{1}{2}\right) .
\end{aligned}
$$

Theorem 23. The number of unstable threshold functions in $\mathcal{F}$ is

$$
2 m n-U(m, n)+8 V\left(\frac{m-1}{2}, \frac{n-1}{2}\right) .
$$

Proof. We first notice that if $u_{x} \leq \frac{m}{2}$ and $u_{y} \leq \frac{n}{2}$, then the number of $u$-stable inclined lines equals $2 U\left(u_{x}, u_{y}\right)$. If $u$ lies on a side of the rectangle $\mathcal{K}$, then the line containing this side is the only $u$-stable line. Otherwise, if $u$ is an internal point of $\mathcal{K}$, then both vertical and horizontal as well as inclined lines passing through $u$ are $u$-stable.

Let us count the number of all $u$-stable lines for $u \in \mathcal{K}_{0}^{\prime}$. Despite that counting depends on the parity of the integers $m$ and $n$, we will show that the result in all cases equals

$$
2(m n-1)+8 V\left(\frac{m-1}{2}, \frac{n-1}{2}\right) .
$$

Below we consider the different possible parities of $m$ and $n$.

If both $m$ and $n$ are odd, then (7) and Lemma 18 imply that the number of all $u$-stable lines for $u=(i, j) \in\left[0, \frac{m-1}{2}\right] \times\left[0, \frac{n-1}{2}\right] \backslash\{0\}$ equals

$$
\sum_{i=1}^{\frac{m-1}{2}} 1+\sum_{j=1}^{\frac{n-1}{2}} 1+\sum_{i=1}^{\frac{m-1}{2}} \sum_{j=1}^{\frac{n-1}{2}}(2 U(i, j)+2)=\frac{m n-1}{2}+2 V\left(\frac{m-1}{2}, \frac{n-1}{2}\right) .
$$


Due to the symmetry, the total number of $u$-stable lines for $u \in \mathcal{K}_{0}^{\prime}$ is four times as many.

Now let $m$ be even and $n$ be odd. Then the number of all $u$-stable lines for $u=(i, j) \in$ $\left[0, \frac{m}{2}-1\right] \times\left[0, \frac{n-1}{2}\right] \backslash\{\mathbf{0}\}$ equals

$$
\sum_{i=1}^{\frac{m}{2}-1} 1+\sum_{j=1}^{\frac{n-1}{2}} 1+\sum_{i=1}^{\frac{m}{2}-1} \sum_{j=1}^{\frac{n-1}{2}}(2 U(i, j)+2)=\frac{m n-n-1}{2}+2 V\left(\frac{m}{2}-1, \frac{n-1}{2}\right) .
$$

Quadruplicated this number counts the $u$-stable lines for $u \in \mathcal{K}_{0}^{\prime}$, except for $u$ lying on the line $x=\frac{m}{2}$. Due to property (8), the $u$-stable lines for $u$ lying on the line $x=\frac{m}{2}$ can be counted as

$$
2\left(1+\sum_{j=1}^{\frac{n-1}{2}}\left(2 U\left(\frac{m}{2}, j\right)+2\right)\right)=2 n+4\left(V\left(\frac{m}{2}, \frac{n-1}{2}\right)-V\left(\frac{m}{2}-1, \frac{n-1}{2}\right)\right) .
$$

Hence, the total number of $u$-stable lines for $u \in \mathcal{K}_{0}^{\prime}$ equals

$$
\begin{aligned}
& 4\left(\frac{m n-n-1}{2}+2 V\left(\frac{m}{2}-1, \frac{n-1}{2}\right)\right)+2 n+4\left(V\left(\frac{m}{2}, \frac{n-1}{2}\right)-V\left(\frac{m}{2}-1, \frac{n-1}{2}\right)\right) \\
& =2(m n-1)+4\left(V\left(\frac{m}{2}, \frac{n-1}{2}\right)+V\left(\frac{m}{2}-1, \frac{n-1}{2}\right)\right)=2(m n-1)+8 V\left(\frac{m-1}{2}, \frac{n-1}{2}\right) .
\end{aligned}
$$

Here we used the property (6).

The case of odd $m$ and even $n$ is considered similarly.

Finally, let both $m$ and $n$ be even. The number of all $u$-stable lines for $u=(i, j) \in$ $\left[0, \frac{m}{2}-1\right] \times\left[0, \frac{n}{2}-1\right] \backslash\{0\}$ equals

$$
\sum_{i=1}^{\frac{m}{2}-1} 1+\sum_{j=1}^{\frac{n}{2}-1} 1+\sum_{i=1}^{\frac{m}{2}-1} \sum_{j=1}^{\frac{n}{2}-1}(2 U(i, j)+2)=\frac{m n-m-n}{2}+2 V\left(\frac{m}{2}-1, \frac{n}{2}-1\right) .
$$

Quadruplicated this number equals the number of all $u$-stable lines for $u \in \mathcal{K}_{0}^{\prime}$, except for those lying on the lines $x=\frac{m}{2}$ or $y=\frac{n}{2}$. Properties (8) and (10) imply that the number of $u$-stable lines for $u$ lying on these lines equals

$$
\begin{aligned}
& 2\left(1+1+\sum_{i=1}^{\frac{m}{2}-1}\left(2 U\left(i, \frac{n}{2}\right)+2\right)+\sum_{j=1}^{\frac{n}{2}-1}\left(2 U\left(\frac{m}{2}, j\right)+2\right)\right)+2 U\left(\frac{m}{2}, \frac{n}{2}\right)+2 \\
& =2(m+n-1)+2 U\left(\frac{m}{2}, \frac{n}{2}\right)+4\left(V\left(\frac{m}{2}-1, \frac{n}{2}\right)+V\left(\frac{m}{2}, \frac{n}{2}-1\right)-2 V\left(\frac{m}{2}-1, \frac{n}{2}-1\right)\right) \\
& =2(m+n-1)+2 V\left(\frac{m-1}{2}, \frac{n-1}{2}\right)-8 V\left(\frac{m}{2}-1, \frac{n}{2}-1\right) .
\end{aligned}
$$

Summing up the results, we get (11).

Lemmas 18 and 20 imply that the total number of unstable threshold functions with the vertex in $\mathcal{K}_{0}^{\prime}$ is equal to (11) minus $U(m, n)-1$. Finally, we need to add 1 for the single unstable threshold function with the vertex at $\mathbf{0}$. 
The number of two-dimensional threshold functions

\subsection{Number of stable threshold functions}

Theorem 24. The number of stable threshold functions in $\mathcal{F}$ equals

$$
m+n+U(m, n)+2 V(m, n)-8 V\left(\frac{m-1}{2}, \frac{n-1}{2}\right) .
$$

Proof. Consider any stable line $\ell$ of a positive slope passing through 0 . Let a point $(a, b) \in l \cap \mathcal{K}_{0}$ be the closest to 0 , implying that $\operatorname{gcd}(a, b)=1$.

Consider all stable lines parallel to $\ell$. Every such line is defined by a pair of points $(x, y),(x+a, y+b) \in \mathcal{K}_{0}$ on it, where $(x, y) \in \mathcal{K}_{0}$ is the closest point to 0 . Such pairs are uniquely defined by the following constraints

$$
\left\{\begin{array}{l}
x<a \text { or } y<b, \\
x+a \leq m, \\
y+b \leq n
\end{array}\right.
$$

Let $P$ be the set of all points $(x, y) \in \mathcal{K}_{0}$ satisfying these constraints. Then the stable lines parallel to $\ell$ and the elements of $P$ are in one-to-one correspondence.

If $a>\frac{m}{2}$ or $b>\frac{n}{2}$, then the set $P$ equals $P_{1} \stackrel{\text { def }}{=}\left\{(x, y) \in \mathcal{K}_{0} \mid 0 \leq x \leq m-a, 0 \leq y \leq n-b\right\}$. In this case the number of stable lines parallel to $\ell$ is $(m+1-a)(n+1-b)$.

If $a \leq \frac{m}{2}$ and $b \leq \frac{n}{2}$, then the set $P$ equals

$$
P_{1} \backslash\left\{(x, y) \in \mathcal{K}_{0} \mid a \leq x \leq m-a, b \leq y \leq n-b\right\} .
$$

Hence, in this case the number of stable lines parallel to $\ell$ is less than before by

$$
(m+1-2 a)(n+1-2 b)=4\left(\frac{m-1}{2}+1-a\right)\left(\frac{n-1}{2}+1-b\right) .
$$

Summing over all pairs $(a, b)$ fulfilling the constraints and using formula (5), we derive that the total number of stable lines of a positive (negative) slope is $V(m, n)-4 V\left(\frac{m-1}{2}, \frac{n-1}{2}\right)$, while the total number of inclined stable lines is twice as many.

Since each inclined stable line passing through $\mathbf{0}$ defines two distinct threshold functions, we further increase the count by the number of such lines, i.e., $U(m, n)$.

Finally, we take into account $m$ vertical lines $x=i$ for $i=\overline{0, m-1}$ and $n$ horizontal lines $y=j$ for $j=\overline{0, n-1}$ to complete the proof.

Adding up the results of Theorems 23 and 24 and noticing that $N(m, n)=2(|\mathcal{F}|+1)$ completes the proof of Theorem 3

\section{Asymptotics of $N(m, n)$}

In this section we prove the following theorem, which together with (3) implies asymptotics (2). 
Theorem 25. For $m \geq n$, the following asymptotics holds

$$
\begin{gathered}
N(m, n)=\frac{6}{\pi^{2}} m^{2} n^{2}+O\left(m^{2} n \ln n\right) \\
N(m, n)=2((n+1) \Psi(n)-\Phi(n)) m^{2}+O\left(m n^{3}\right)
\end{gathered}
$$

where $\varphi(t)$ is the totient function and

- $\Phi(k) \stackrel{\text { def }}{=} \sum_{i=1}^{k} \varphi(i)=\frac{3}{\pi^{2}} k^{2}+O(k \ln k)$ (Dirichlet's Theorem, see [9]);

- $\Psi(k) \stackrel{\text { def }}{=} \sum_{i=1}^{k} \frac{\varphi(i)}{i}=\frac{6}{\pi^{2}} k+O(\ln k)($ see [9]).

We remark that the first of the two asymptotics for $N(m, n)$ given in Theorem 25is more suitable for $m$ and $n$ of the same magnitude, while the second asymptotics is better when $m \gg n$.

Lemma 26. Let $k$ be a positive integer and $s \geq 0$. Then

$$
\begin{gathered}
\sum_{t=1}^{k} \frac{1}{t}=O(\ln k) \\
\sum_{t=k+1}^{\infty} \frac{1}{t^{s}}=O\left(\frac{1}{k^{s-1}}\right), \quad(s \neq 1) \\
\sum_{t=1}^{k} t^{s} \ln t=O\left(k^{s+1} \ln k\right) \\
\sum_{t=1}^{k} t^{s}=\frac{k^{s+1}}{s+1}+O\left(k^{s}\right)=O\left(k^{s+1}\right) .
\end{gathered}
$$

Proof. The statement follows from integral estimates of the sums.

Theorem 27. For $t \geq k$, the following inequality holds

$$
U(t, k)=U(k, t)=\frac{6}{\pi^{2}} t k+O(t \ln k) ;
$$

Proof. Note that there exist exactly $\lfloor m / p\rfloor$ positive integers not exceeding $m$ that are divisible by $p$. Hence, there are $\lfloor t / p\rfloor\lfloor k / p\rfloor$ pairs $(a, b), 1 \leq a \leq t, 1 \leq b \leq k$, whose greatest common divisor is divisible by $p$. The inclusion-exclusion principle [13] for the number of pairs $(a, b)$ with $\operatorname{gcd}(a, b)$ not divisible by any prime $p($ i.e., $\operatorname{gcd}(a, b)=1)$ gives an exact formula

$$
U(t, k)=\sum_{s=1}^{\infty} \mu(s)\left\lfloor\frac{t}{s}\right\rfloor\left\lfloor\frac{k}{s}\right\rfloor,
$$


The number of two-dimensional threshold functions

where $\mu(s)$ is the Möbeus function.

We approximate $U(t, k)$ with the function

$$
\hat{U}(t, k) \stackrel{\text { def }}{=} \sum_{s=1}^{\infty} \mu(s) \frac{t k}{s} \frac{k}{s}=t k \sum_{s=1}^{\infty} \frac{\mu(s)}{s^{2}}=t k \frac{6}{\pi^{2}} .
$$

and bound the absolute value of the difference $U(t, k)-\hat{U}(t, k)$ as follows

$$
\left.|U(t, k)-\hat{U}(t, k)|=\mid \sum_{s=1}^{\infty} \mu(s)\left(\left\lfloor\frac{t}{s}\right\rfloor \mid \frac{k}{s}\right\rfloor-\frac{t}{s} \frac{k}{s}\right) \mid \leq \sum_{s=1}^{\infty} d(t, k, s),
$$

where

$$
d(t, k, s) \stackrel{\text { def }}{=} \frac{t k}{s^{2}}-\left\lfloor\frac{t}{s}\right\rfloor\left\lfloor\frac{k}{s}\right\rfloor .
$$

For $s>k$, we have $\left\lfloor\frac{k}{s}\right\rfloor=0$ and thus $d(t, k, s)=\frac{t k}{s^{2}}$. For $s \leq k$, we bound $d(t, k, s)$ as follows:

$$
d(t, k, s)<\frac{t k}{s^{2}}-\left(\frac{t}{s}-1\right)\left(\frac{k}{s}-1\right)=\frac{t+k}{s}-1<\frac{2 t}{s} .
$$

Applying Lemma 26, we finally have

$$
|U(t, k)-\hat{U}(t, k)|<\sum_{s=1}^{\infty} d(t, k, s)<\sum_{s=1}^{k} \frac{2 t}{s}+\sum_{s=k+1}^{\infty} \frac{t k}{s^{2}}=O(t \ln k) .
$$

We remark that Dirichlet's Theorem is a particular case of Theorem 27 for $t=k$.

Theorem 28. For $t \geq k$, the following asymptotics holds

$$
V(t, k)=V(k, t)=\frac{3}{2 \pi^{2}} t^{2} k^{2}+O\left(t^{2} k \ln k\right) .
$$

Proof. We use formula (7) and property $U(i, j)=U(j, i)$ as follows:

$$
V(t, k)=\sum_{i=1}^{t} \sum_{j=1}^{k} U(i, j)=2 \sum_{i=1}^{k} \sum_{j=1}^{i-1} U(i, j)+\sum_{i=1}^{k} U(i, i)+\sum_{i=k+1}^{t} \sum_{j=1}^{k} U(i, j) .
$$

According to Lemmas 26 and 27 .

$$
\begin{aligned}
& \sum_{i=1}^{k} \sum_{j=1}^{i-1} U(i, j)=\sum_{i=1}^{k} \sum_{j=1}^{i-1}\left(\frac{6}{\pi^{2}} i j+O(i \ln j)\right)=\frac{6}{\pi^{2}} \sum_{i=1}^{k}\left(i \sum_{j=1}^{i-1} j+O\left(i \sum_{j=1}^{i-1} \ln j\right)\right)= \\
& =\frac{6}{\pi^{2}} \sum_{i=1}^{k}\left(i^{i^{2}}+O\left(i^{2} \ln i\right)\right)=\frac{3}{\pi^{2}} \sum_{i=1}^{k} i^{3}+O\left(\sum_{i=1}^{k} i^{2} \ln i\right)=\frac{3}{4 \pi^{2}} k^{4}+O\left(k^{3} \ln k\right) .
\end{aligned}
$$


Similarly,

$$
\sum_{i=1}^{k} U(i, i)=\sum_{i=1}^{k} O\left(i^{2}\right)=O\left(k^{3}\right)
$$

and

$$
\begin{aligned}
\sum_{i=k+1}^{t} \sum_{j=1}^{k} U(i, j) & =\sum_{i=k+1}^{t} \sum_{j=1}^{k}\left(\frac{6}{\pi^{2}} i j+O(i \ln j)\right)=\sum_{i=k+1}^{t}\left(\frac{6}{\pi^{2}} i \frac{k^{2}}{2}+O(i k \ln k)\right) \\
& =\frac{3}{2 \pi^{2}} k^{2}\left(t^{2}-k^{2}\right)+O\left(t^{2} k \ln k\right) .
\end{aligned}
$$

Therefore,

$$
\begin{aligned}
V(t, k) & =2\left(\frac{3}{4 \pi^{2}} k^{4}+O\left(k^{3} \ln k\right)\right)+O\left(k^{3}\right)+\frac{3}{2 \pi^{2}} k^{2}\left(t^{2}-k^{2}\right)+O\left(t^{2} k \ln k\right) \\
& =\frac{3}{2 \pi^{2}} t^{2} k^{2}+O\left(t^{2} k \ln k\right)
\end{aligned}
$$

Theorem 29. For $t \geq k$, the following asymptotics holds

$$
U(t, k)=U(k, t)=\Psi(k) t+O\left(k^{2}\right) .
$$

Proof. We use formula (4)

$$
\begin{aligned}
U(k, t) & =\sum_{\substack{i=1 \\
\operatorname{gcd}(i, j)=1}}^{k} \sum_{j=1}^{t} 1=\sum_{i=1}^{k}\left(\sum_{s=0}^{\left\lfloor\frac{t}{i}\right\rfloor-1} \sum_{\substack{j=s i+1 \\
\operatorname{gcd}(j, i)=1}}^{s i+i} 1+\sum_{\substack{j=\lfloor t / j i j+1 \\
\operatorname{gcd}(j, i)=1}}^{t} 1\right)=\sum_{i=1}^{k}\left\lfloor\frac{t}{i}\right\rfloor \varphi(i)+O\left(k^{2}\right)=\sum_{i=1}^{k} \frac{t}{i} \varphi(i)+O\left(k^{2}\right) \\
& =\Psi(k) t+O\left(k^{2}\right) .
\end{aligned}
$$

Theorem 30. For $t \geq k$, the following asymptotics holds

$$
V(t, k)=V(k, t)=\frac{(k+1) \Psi(k)-\Phi(k)}{2} t^{2}+O\left(t k^{3}\right) .
$$

Proof. We use formula (5)

$$
\begin{aligned}
V(k, t) & =\sum_{\substack{i=1 \\
\operatorname{gcd}(i, j)=1}}^{k} \sum_{j=1}^{t}(k+1-i)(t+1-j) \\
& =\sum_{i=1}^{k}(k+1-i)\left(\sum_{s=0}^{\left\lfloor\frac{t}{i}\right\rfloor-1} \sum_{\substack{j=1 \\
\operatorname{gcd}(j, i)=1}}^{i}(t+1-s i-j)+\sum_{\substack{j=1 \\
\operatorname{gcd}(j, i)=1}}^{t \bmod i}(t \bmod i+1-j)\right) .
\end{aligned}
$$


Neglecting terms of order $O\left(t k^{3}\right)$, we have

$$
\begin{aligned}
V(k, t) & =\sum_{i=1}^{k}(k+1-i) \sum_{s=0}^{\left\lfloor\frac{t}{i}\right\rfloor-1} \sum_{\substack{j=1 \\
\operatorname{gcd}(j, i)=1}}^{i}(t-s i)+O\left(t k^{3}\right)=\frac{t^{2}}{2} \sum_{i=1}^{k}(k+1-i) \frac{\varphi(i)}{i}+O\left(t k^{3}\right) \\
& =\frac{(k+1) \Psi(k)-\Phi(k)}{2} t^{2}+O\left(t k^{3}\right) .
\end{aligned}
$$

Theorem 25 now follows from Theorems 3, 28, and 30 ,

\section{Relation to teaching sets}

A teaching set [2, 11, 15] of a threshold function $f: \mathcal{K}_{0} \rightarrow\{0,1\}$ is a subset $T \subset \mathcal{K}_{0}$ such that for any other threshold function $g \neq f$, there exists $t \in T$ with $g(t) \neq f(t)$. A teaching set of minimal cardinality is called minimum teaching set. A minimum teaching set of a two-dimensional threshold function consists of either 3 or 4 points [11].

It is easy to see that any unstable threshold function $f \in \mathcal{F}$ has a teaching set of size 3 . Indeed, let $u \in \mathcal{K}_{0}$ be the vertex of $f$ and $\ell \ni u$ be a line defining $f$. Then $\ell$ lies between two adjacent stable lines $\ell_{1}$ and $\ell_{2}$ passing respectively through some points $u_{1} \neq u$ and $u_{2} \neq u$ with $f\left(u_{1}\right)=f\left(u_{2}\right) \neq f(u)$. Then $\left\{u, u_{1}, u_{2}\right\}$ forms a teaching set of $f$.

However, the size of a minimum teaching set $T$ of a stable threshold function $f \in \mathcal{F}$ may be 3 or 4 . Namely, $|T|=3$ if the complement threshold function $\tilde{f}(x, y)=1-f(m-x, n-y)$ is unstable; and $|T|=4$ if $\tilde{f}(x, y)$ is stable. Therefore, the stable threshold functions can be partitioned into two classes, depending on the size of their minimal teaching sets. Unfortunately, our results do not allow to compute the number of threshold functions in each class, which we pose as an open problem.

\section{Acknowledgements}

The author thanks Nikolai Zolotykh and Valeriy Shevchenko for posing the problem of counting threshold functions and related invaluable discussions, and Keith Conrad for reviewing an earlier version of the paper.

\section{References}

[1] H. Abelson, Computational geometry of linear threshold functions, Information and Control, 34 (1977), pp. 66-92.

[2] S. A. Goldman and M. J. Kearns, On the Complexity of Teaching, Journal of Computer and System Sciences, 50 (1995), pp. 20-31.

[3] M. H. Hassoun, Fundamentals of Artificial Neural Networks, MIT Press, 1995. 
The number of two-dimensional threshold functions

[4] A. A. Irmatov, Arrangements of Hyperplanes and the Number of Threshold Functions, Acta Applicandae Mathematicae, 68 (2001), pp. 211-226.

[5] A. A. Irmatov and Z. D. Kovijanic, On the asymptotics of the logarithm of the number of threshold functions in k-valued logic, Discrete Mathematics and Applications, 8 (1998), pp. 331-355.

[6] S. Muroga, Threshold Logic and Its Applications, Published by Wiley-Interscience, 1971.

[7] S. Muroga, T. Tsuboi, and C. R. Baugh, Enumeration of threshold functions of eight variables, IEEE Transactions on Computers, C-19 (1970), pp. 818-825.

[8] P. С. Ојна, Enumeration of linear threshold functions from the lattice of hyperplane intersections, IEEE Transactions on Neural Networks, 11 (2000), pp. 839-850.

[9] K. Prachar, Primzahlverteilung, Springer-Verlag, 1957.

[10] V. N. Shevchenko, Qualitative Topics in Integer Linear Programming, American Mathematical Society, 1997.

[11] V. N. Shevchenko and N. Yu. Zоцотүкн, On the complexity of deciphering the threshold functions of k-valued logic, Dokl. Math., 58 (1998), pp. 268-270.

[12] N. J. A. Sloane, On-Line Encyclopedia of Integer Sequences, published electronically http://oeis.org. (2010).

[13] R. Stanley, Enumerative combinatorics, vol. 1, Wadsworth \& Brooks/Cole, 1986.

[14] R. O. WINDER, Enumeration of seven-argument threshold functions, IEEE Transactions on Electronic Computers, EC-14 (1965), pp. 315-325.

[15] N. Yu. ZоLотүкн and V. N. Shevchenкo, Estimating the complexity of deciphering a threshold functions in a $k$-valued logic, Computational mathematics and mathematical physics, 39 (1999), pp. 328-334.

[16] Yu. A. Zuev, Asymptotics of the logarithm of the number of threshold functions of the algebra of logic, Sov. Math., Dokl., 39 (1989), pp. 512-513.

[17] Combinatorial-probabilistic and geometric methods in threshold logic, Diskretn. Mat., 3 (1991), pp. 47-57.

[18] J. Zunic, On encoding and enumerating threshold functions, IEEE Transactions on Neural Networks, 15 (2004), pp. 261-267. 